\title{
Top Quark Production at CDF
}

\author{
Shulamit Moed \\ on behalf of the CDF collaboration \\ Harvard University \\ Laboratory for Particle Physics and Cosmology \\ 18 Hammond St. \\ Cambridge MA 02138 \\ email: moed@physics.harvard.edu
}

\begin{abstract}
.
The large data samples of top quark candidate events collected at the Tevatron CDF II experiment allow for a variety of measurements to analyze the production of the top quark. This article discusses recent results of top quark production at CDF presented at the SUSY09 conference, including updates to the top pair production cross section, forward-backward asymmetry in $t \bar{t}$ production, single top search, search for top resonances and a search for heavy top. The discussed measurements utilize up to $3.2 \mathrm{fb}^{-1}$ of integrated luminosity collected at CDF.
\end{abstract}

Keywords: CDF, Tevatron, top, production

PACS: $14.65 .-\mathrm{q}, 14.65 . \mathrm{Ha}$

\section{INTRODUCTION}

At the Tevatron collider at the Fermi National Accelerator Laboratory, protons and antiprotons are collided at a center-of-mass energy of $1.96 \mathrm{TeV}$. Providing the highest energies currently available at a hadron collider, the Tevatron is still the only place where top quarks are produced. CDF has extensively studied the production and decays of the top quark, as precision measurements have the potential to reveal new physics effects. The focus of this article is on measurements of top quark production $(t \bar{t}$ and single top), forward-backward asymmetry in top pair production, and searches for new physics effects in top resonances and heavy top quark production. Events in the analyses presented here are selected in the lepton+jets channel.

\section{MEASUREMENTS}

\section{Top Pair Production Cross Section}

The SM top quark at the Tevatron is mostly produced in pairs with a theoretical cross section prediction of $6.7 \pm 0.8 \mathrm{pb}$ [1]. The top quark pair production cross section measurement is an important test of QCD predictions and is the key to all top property measurements, since it requires the understanding of all background processes in the selected sample of candidate top quark events. In the lepton+jets channel of the $t \bar{t}$ decay the dominant backgrounds come from $\mathrm{W}$ boson production in association 
with jets, and non-W QCD events. Other backgrounds which may have similar final states are di-boson, single top and $Z \rightarrow \tau \tau$ processes. The backgrounds are estimated using a combination of data and Monte Carlo methods. The excess in data over these backgrounds is attributed to top pair production. Signal to background discrimination is improved by using a secondary vertex finding algorithm to reconstruct heavy flavor decay vertices inside jets ('b-tagging'). At least one of the jets is required to be tagged as a b-jet. To further suppress the background, the scalar sum of all transverse energies in the event, $H_{T}$, is required to exceed $250 \mathrm{GeV}$. Using $3.2 \mathrm{fb}^{-1}$ of integrated luminosity, the top pair production cross-section is measured to be $7.2 \pm 0.4($ stat $) \pm 0.5($ syst $) \pm$ 0.4 (lumi $) \mathrm{pb}$. Another measurement uses a neural network technique to discriminate between top pair production and background processes in the same data sample and measures this cross section to be $7.0 \pm 0.4$ (stat) \pm 0.4 (syst) \pm 0.4 (lumi) pb.

The dependence on the luminosity measurement and its associated large systematic uncertainty is significantly reduced by computing the ratio of the $t \bar{t}$ to $\mathrm{Z}$ cross section, measured using the same triggers and dataset. This ratio is then multiplied by the theoretical $Z$ cross section. The final $t \bar{t}$ cross section, assuming a top mass of 175 $\mathrm{GeV} / c^{2}$, is measured to $7.0 \pm 0.4$ (stat) \pm 0.6 (syst \pm 0.1 (theory) pb using the secondary vertex finding method, and $6.8 \pm 0.4$ (stat) \pm 0.4 (syst $) \pm 0.1$ (theory) pb using the neural network technique.

\section{Single Top Quark Production}

Several searches for single top quark production have been performed at CDF using a variety of techniques such as neural network and boosted decision trees. These analyses are combined in a procedure using evolved neural networks (NEAT). This procedure gets as an input the discriminants of the different individual analyses on an event by event basis and outputs a "superdiscriminant" for each event. An analysis that looks for a signature of missing transverse energy + jets, unlike the other techniques that look for a charged lepton + missing energy + jets is combined with the superdiscriminant from the other measurements later. For the s-channel (time-like production in Feynman diagram) and t-channel (space-like production in Feynman diagram) search the combination achieves an expected $\mathrm{p}$-value of $5.9 \sigma$. This p-value is the probability of making an observation as significant as this one, assuming no single top production. The observed p-value is $5.0 \sigma$, The observed p-value of $5.0 \sigma$ leads us to conclude that we have observed single top quark production. A fit for the combined $s+t$ channel single top quark production rate yields a cross-section of $2.3_{-0.5}^{+0.6} \mathrm{pb}$. Because the cross-section is directly proportional to the CKM matrix element $V_{t b}$, the strength of the $V_{t b}$ vertex can be extracted from the measured cross section. The CKM matrix element $V_{t b}$ is measured $\left|V_{t b}\right|=0.91 \pm 0.11$ (experiment) \pm 0.07 (theory). The theoretical uncertainties arise from the cross section dependence on the top quark mass, parton distribution functions, factorization and renormalization scales, and $\alpha_{s}$. This observation is described in detail in [2]. 


\section{Forward-Backward Asymmetry in $t \bar{t}$ Events}

Several theories beyond the SM predict a sizable forward backward asymmetry. In addition, QCD at NLO predicts a non-zero asymmetry in $q \bar{q} \rightarrow t \bar{t}$ events [3]. Because top quark pair production will be dominated by gluon fusion at the LHC, the Tevatron is a unique place to study these effects where the top pairs are mostly produced via $q \bar{q}$ annihilation. Two independent techniques were applied in this measurement, each in a different center-of-mass frame: $p \bar{p}$ and $t \bar{t}$. The two frames differ in that the measurement in the $p \bar{p}$ frame is less sensitive to bias or dilution from the experiment, and that in the $t \bar{t}$ frame is more sensitive to the QCD predicted asymmetry. A model independent unfolding is used in the $p \bar{p}$ frame and a likelihood fit to a linear asymmetry in the production angle $(1+A \cos \alpha)$ is used in the $t \bar{t}$ frame. The measured asymmetry with $3.2 f b^{-1}$ is $A_{f b}=0.193 \pm 0.065$ (stat) \pm 0.024 (syst), compared to the small charge asymmetry expected in QCD at NLO, $A_{f b}=0.05 \pm 0.015$.

\section{Search for Heavy Top-like Quarks}

Using $2.8 \mathrm{fb}^{-1}$ of integrated luminosity CDF searches for pair production of a new heavy top-like quark $\left(t^{\prime}\right)$. A forth chiral generation of massive fermions is predicted in a number of models, such as two-Higgs-doublet scenarios and N=2 SUSY models [4]. A small mass splitting between new heavy $t^{\prime}$ and $b^{\prime}$ quarks is preferred, thus $t^{\prime}$ decays predominantly to a $W$ boson and a down-type quark $(\mathrm{q}=\mathrm{d}, \mathrm{s}, \mathrm{b})$. Such heavy exotic quarks are present in other models as well, like the beautiful mirrors [5], while a heavy top quark also appears in little Higgs models [6]. Assuming this new quark is pair-produced strongly, has a mass greater than the top quark mass and decays predominantly to $W q$, its event signature would mimic that of the top pair production. To distinguish the $t^{\prime}$ signal from the SM backgrounds, a 2D fit to the $t^{\prime}$ reconstructed mass and the scalar sum of the total transverse energy $\left(H_{T}\right)$ is performed. No evidence of $t^{\prime}$ is observed and the $95 \%$ CL upper limit on the $t^{\prime}$ production rate is set, excluding a $t^{\prime}$ quark with a mass below $311 \mathrm{Gev} / c^{2}$ when comparing with theoretical prediction [7], as shown in Figure 1.

\section{Measurement of the $t \bar{t}$ differential cross section, $d \sigma / d M_{t \bar{t}}$}

In several models of beyond the SM physics gauge interactions can produce massive particles which may couple strongly to the top quark. Such particles may produce resonances in the invariant mass distribution of the top quark via a $p \bar{p} \rightarrow X \rightarrow t \bar{t}$ process, or may interfere with the SM process and cause distortions to the shape of the invariant mass distribution [8]. The consistency of this measurement with the SM is established by comparing the value of the Anderson-Darling test statistic observed in the data and in SM-only pseudo-experiments from Monte carlo simulations. The p-value for this measurement in the SM-only hypothesis is $34 \%$. A limit is then set on the $\kappa / M_{p l}$ in the RS model of $\kappa / M_{p l}>0.16$ at $95 \%$ CL by studying the coupling of KK gravitons to top quarks, where the mass of the first resonance is $600 \mathrm{GeV} / c^{2}$. 
FIGURE 1. Upper limit, at $95 \% \mathrm{CL}$, on the production rate for t' as a function of the t' mass (red). the purple curve is the theoretical cross-section. The dark (light) blue band represents the $\pm 1 \sigma( \pm 2 \sigma)$ contour on the expected rate.

\section{CONCLUSIONS}

CDF has an ongoing rich program of top quark production and property analyses, which all become high precision measurements as the top quark candidate data samples keep getting larger. No evidence for New Physics have been found with the current datasets, however, some of these measurement provide constraints on several beyond the Standard Model theories.

\section{ACKNOWLEDGMENTS}

I thank the members of the CDF experiment, and in particular the CDF top group and authors of the mentioned analyses.

\section{REFERENCES}

1. M. Cacciari et al., JHEP 404, 68 (2004)

2. T. Aaltonen et al., The CDF Collaboration,Phys. Rev. Lett. 103, 092002 (2009). arXiv: 0903.0885

3. J.H. Kuhn and G. Rodrigo. Phys. Rev. Lett. 81, 49 (1998)

4. Hong-Jian He et al. Phys. Rev. D, 64(2001) 053004

5. D. Choudhury et al. Phys. Rev. D, 65(2002) 053002

6. M. Perelstien et al. Phys. Rev. D, 69(2004) 075002

7. R. Bonciani et al. Nucl. Phys., 529(1998) 424

8. Christopher T. Hill and Stephen J. Parke Phys. Rev. D 494454 (1994); D. Dicus, A. Stange, S. Willenbrock, Phy. Lett. S; G. Cvetic, Reviews of Modern Physics 71513 (1999); R. Frederix and F. Maltoni “Top Pai Invariant Mass Ditribution: a Window on New Physcis” (2007) hep-ph/07122355v1 